\title{
ANALYSis OF THE First COLIBRI Fuel Rods OSCILlation CAMPAign IN THE CROCUS REACTOR FOR THE EUROPEAN PROJECT CORTEX
}

\author{
V. Lamirand ${ }^{1,2}$, A. Rais ${ }^{1}$, O. Pakari ${ }^{1}$, M. Hursin ${ }^{1,2}$, A. Laureau ${ }^{1}$, J. Pohlus ${ }^{4}$, U. Paquee ${ }^{4}$, \\ C. Pohl ${ }^{5}$, S. Hübner ${ }^{3}$, C. Lange ${ }^{3}$, P. Frajtag ${ }^{1}$, D. Godat ${ }^{1}$, G. Perret ${ }^{2}$, C. Fiorina ${ }^{1}$, A. Pautz ${ }^{1,2}$ \\ ${ }^{1}$ Ecole Polytechnique Fédérale de Lausanne (EPFL), 1015 Lausanne, Switzerland \\ ${ }^{2}$ Paul Scherrer Institut (PSI), 5232 Villigen, Switzerland \\ ${ }^{3}$ Technische Universität Dresden (TUD), 01062 Dresden, Germany \\ ${ }^{4}$ Institut fur Sicherheitstechnologie GmbH (ISTec), 85748 Garching, Germany \\ ${ }^{5}$ TÜV Rheinland Industrie Service GmbH (TUV), 51105 Cologne, Germany \\ vincent.lamirand@epfl.ch
}

\begin{abstract}
The Horizon2020 European project CORTEX aims at developing an innovative core monitoring technique that allows detecting anomalies in nuclear reactors, such as excessive vibrations of core internals, flow blockage, or coolant inlet perturbations. The technique will be mainly based on using the fluctuations in neutron flux recorded by in-core and ex-core instrumentation, from which the anomalies will be differentiated depending on their type, location and characteristics. The project will result in a deepened understanding of the physical processes involved, allowing utilities to detect operational problems at a very early stage. In this framework, neutron noise computational methods and models are developed. In parallel, mechanical noise experimental campaigns are carried out in two zero-power reactors: AKR-2 and CROCUS. The aim is to produce high quality neutron noise-specific experimental data for the validation of the models. In CROCUS, the COLIBRI experimental program was developed to investigate experimentally the radiation noise induced by fuel rods vibrations. In this way, the 2018 first CORTEX campaign in CROCUS consisted in experiments with a perturbation induced by a fuel rods oscillator. Eighteen fuel rods located at the periphery of the core fuel lattice were oscillated between $\pm 0.5 \mathrm{~mm}$ and $\pm 2.0 \mathrm{~mm}$ around their central position at a frequency ranging from $0.1 \mathrm{~Hz}$ to $2 \mathrm{~Hz}$. Signals from 11 neutron detectors which were set at positions in-core and ex-core in the water reflector, were recorded. The present article documents the results in noise level of the experimental campaign. Neutron noise levels are compared for several oscillation frequencies and amplitudes, and at the various detector locations concluding to the observation of a spatial dependency of the noise in amplitude.
\end{abstract}

KEYWORDS: Core monitoring, noise analysis, neutron noise, fuel rod vibration, research reactor experiment, zero-power reactor, CROCUS, CORTEX 


\section{INTRODUCTION}

The Horizon 2020 European project CORTEX aims at developing innovative core monitoring techniques that allow detecting anomalies in nuclear reactors, such as excessive vibrations of core internals, flow blockage, or coolant inlet perturbations [1]. The technique will be mainly based on using the inherent fluctuations in neutron flux recorded by in-core and ex-core instrumentation, from which the anomalies will be differentiated depending on their type, location and characteristics. The method is non-intrusive and does not require any external perturbation of the system. The project will result in a deepened understanding of the physical processes involved. This will allow utilities to detect operational problems at a very early stage, and to take proper actions before such problems have any adverse effect on plant safety and reliability.

In this framework, computer methods and models are developed. The purpose of the experimental campaigns at the AKR-2 and CROCUS reactors is to produce high quality noise-specific experimental data for the validation of these neutron noise computational models. The first campaigns at both facilities consisted in measurements at reference static states, and with the addition of mechanical perturbations [2].

The CROCUS reactor at the École Polytechnique Fédérale de Lausanne (EPFL) is a two-zone, uraniumfueled, and light water-moderated facility. With a maximum power of $100 \mathrm{~W}$, it is a zero-power reactor used for teaching and research purposes, currently with a focus on studies on intrinsic and induced neutron noise [3]-[5]. In CROCUS, the project benefited from the COLIBRI experimental program: up to 18 periphery fuel rods are oscillated radially with sinusoidal motion at a maximum of $\pm 2.5 \mathrm{~mm}$ around their central position in the $\mathrm{Hz}$ range. The present article documents the results in neutron noise levels. In Section 2, the setup and experiments of the first campaign are briefly described. In Section 3, the methodology and results are presented, with regards to the general behavior of the induced noise with perturbation's amplitude and frequency, and the observation of a spatial dependency.

\section{DESCRIPTION OF THE EXPERIMENTAL CAMPAIGN}

The first experimental campaign for CORTEX in the CROCUS reactor took place from 17 to 21 September 2018. Vibrating fuel rods experiments were carried out using an in-core device that was specifically developed for that purpose [6]. This section briefly describes the CROCUS reactor, the experimental setup, and the vibrating fuel rods experiments. A more detailed description is provided in [2].

\subsection{The CROCUS reactor}

CROCUS at the École Polytechnique Fédérale de Lausanne (EPFL) is an experimental zero-power reactor, uranium-fueled and light water-moderated, dedicated to research and teaching radiation and reactor physics. A complete description of the reference core can be found in the International Reactor Physics Experiments Handbook (IRPhE) [7], [8]. It has been licensed for operating at a maximum power of $100 \mathrm{~W}$, i.e. a total neutron flux of $\sim 2.5 \cdot 10^{9} \mathrm{~cm}^{-2} \cdot \mathrm{s}^{-1}$ at the core center. Criticality is controlled either by water level using a spillway, or by two $\mathrm{B}_{4} \mathrm{C}$ absorber control rods, with an accuracy of $\pm 0.1 \mathrm{~mm}$ (equivalent to approximately $\pm 0.4 \mathrm{pcm})$ and $\pm 0.5 \mathrm{~mm}( \pm 0.2 \mathrm{pcm})$, respectively. CROCUS operates at room temperature using a controlled water loop with secondary and tertiary circuits, two heat exchangers and an electrical heater. 

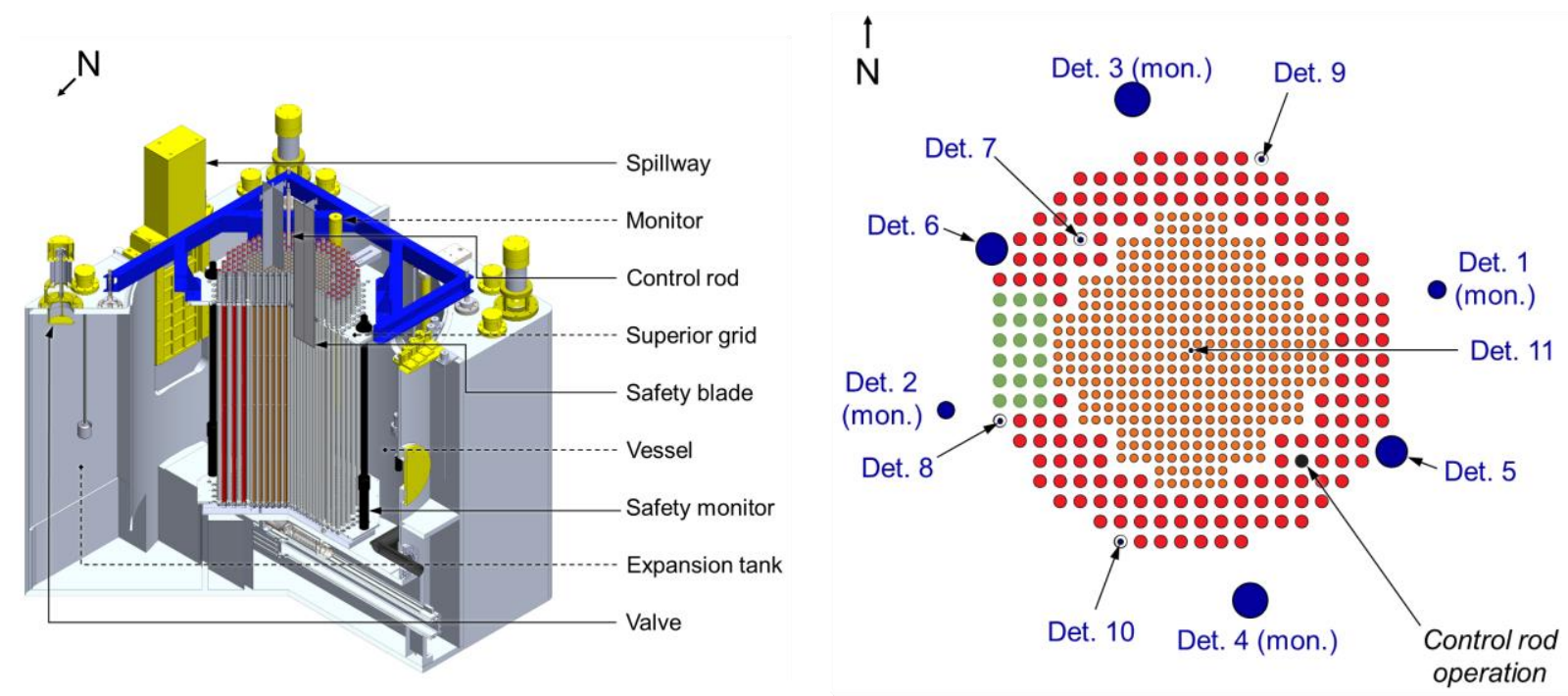

Figure 1. View of the vessel of the CROCUS reactor (left), and top view of the campaign configuration (right) with location of perturbation (green), neutron detectors (blue), and control rod (black).

The core is located in an Al-6060 grade vessel of $130 \mathrm{~cm}$ in diameter, $160 \mathrm{~cm}$ in height, and $1.2 \mathrm{~cm}$ in thickness. The vessel is filled with demineralized light water used as both moderator and reflector. The core active part has the approximate shape of a cylinder of $100 \mathrm{~cm}$ in height and about $60 \mathrm{~cm}$ in diameter. It consists of two interlocked fuel zones with square lattices of different pitches: an inner zone of $336 \mathrm{UO}_{2}$ rods with an enrichment of $1.806 \mathrm{wt} \%$ and a pitch of $1.837 \mathrm{~cm}$; an outer zone of $176 \mathrm{U}_{\text {metal }}$ rods for these experiments, $0.947 \mathrm{wt} . \%$ and $2.917 \mathrm{~cm}$; a varying water gap between the two zones because of the two different pitches. The picture of the reactor and critical assembly configuration is shown in Figure 1.

Both uranium fuels consist of a $1-\mathrm{m}$ pile of cylindrical pellets cladded in aluminum. The rods are maintained vertically by two octagonal aluminum grid plates spaced $1 \mathrm{~m}$ apart. In the COLIBRI program, the grids have a $1 \mathrm{~mm}$ cadmium layer to limit axial neutron leakage to the environment, i.e. structures activation, with the active zone of the fuel starting in the middle of the lower cadmium layer.

\subsection{Experimental setup}

The COLIBRI fuel rods oscillator allows oscillating simultaneously any of 18 metallic uranium fuel rods laterally in the west region of the core periphery zone (see Figure 1, right). It consists of two moving plates set above and below the core grids, and rigidly connected by an aluminum beam. Each plate carries an extremity of the fuel rods, top and bottom respectively. Its oscillation is produced by a motor at the top, and transferred to the bottom moving plate via the aluminum beam. The amplitude of the oscillation is precisely tuned with plates, $0.5 \mathrm{~mm}$ by $0.5 \mathrm{~mm}$ from 0 to $\pm 2.5 \mathrm{~mm}$, and the frequency, set by changing the motor speed, can be set at a maximum of $2 \mathrm{~Hz}$ for safety reasons. The static reactivity worth of the 18 rods was measured at about 10 cents in total for a maximal amplitude of $\pm 2.5 \mathrm{~mm}$.

The sinusoidal oscillation is controlled and monitored via a LabVIEW-developed software, which allows synchronization with the detection instrumentation. An inductive captor monitors the movement of the motor, whereas a cable coder is used to measure the displacement of the bottom moving plate. The oscillator's behavior has been characterized empty, with 1 rod and 18 rods loaded [9]. It is accurate in frequency. In amplitude, the device is rigid at the top, whereas at the bottom it demonstrates inertia inducing an increase of the amplitude for the full 18-rods load case when the frequency is above $1 \mathrm{~Hz}$. 
The detection instrumentation comprised the facility monitors, and different types of additional ex-core and in-core detectors, for a total of eleven detectors of various sizes and sensitivities. The location of each of them with respect to the core and COLIBRI is presented in Figure 1 (right). All four monitor detectors are set at reference positions, as presented in the CROCUS benchmark [7], [8]. The other detectors were set vertically at core mid-height. On the horizontal plane, large fission chambers were set in the reflector west (close to COLIBRI) and east from the core. $\mathrm{BF}_{3}$ counters were set at positions within the lattice as presented in Figure 1, in the NW control rod guide tube (detector 3), and in aluminum channels for the others. The miniature fission chamber was set at core center. The uncertainty on detector position is $\pm 1 \mathrm{~mm}$.

The operation ionization chambers and the large fission chambers were operated in current mode [3], whereas all the other detectors were operated in pulse mode using spectrometry charge preamplifiers. The detectors and their electronics were connected to ISTec, TUD, and both EPFL pulse and current mode instrumentations, with a varying number of detectors depending on the number of available inputs [10]. In addition, the safety monitors were used in MCS mode for power monitoring.

\subsection{Experiments}

Two types of measurements were carried out: static, and a range of measurements in oscillation. The reactor was operated with the south control rod (see Figure 1), at a fixed water level of $1000 \pm 0.1 \mathrm{~mm}$. Following linearity tests, the power was set around $100 \mathrm{~mW}$, and the water temperature was controlled at $20.0 \pm 0.1^{\circ} \mathrm{C}$. In all cases, the 18 rods were lifted in the oscillation position: in the static case, the position was set at zero, i.e. the rods were in their nominal position within the lattice. The global reactivity effect of the oscillations was compensated to ensure a stable power. In practice, the oscillation was started first, then the reactor was stabilized in power with the control rod before starting the measurement. The control rod insertion was updated along the operation and measurement.

The experiments are listed in Table I. Twenty measurements were carried out in total, with limited repetition. All measurements were acquired by all four acquisition systems. Each measurement was chosen to cover enough cycles of the perturbation oscillation, around 1000 cycles except for $0.1 \mathrm{~Hz}$, and last at least $30 \mathrm{~min}$ for resolving the zero-power noise. The amplitude and frequency values listed in Table I are requested values. There is a slight difference between requested and measured values due to the system limitations [9]. In addition to the neutron detection signals, three other outputs were recorded:

- the synchronized signal from the inductive captor,

- the oscillator's structured output, including motor, inductive captor, and cable, and

- the two power monitors outputs.

Table I. Parameters in amplitude, frequency and duration of the oscillation experiments. The asterisk (*) signifies that some repetition was carried out.

\begin{tabular}{|c|c|c|c|c|c|}
\hline \multirow{2}{*}{$\begin{array}{c}\text { Amplitude } \\
(\mathrm{mm})\end{array}$} & \multicolumn{5}{|c|}{ Measurement duration for each frequency (min) } \\
\cline { 2 - 6 } & $0.1 \mathrm{~Hz}$ & $0.5 \mathrm{~Hz}$ & $1.0 \mathrm{~Hz}$ & $1.5 \mathrm{~Hz}$ & $2.0 \mathrm{~Hz}$ \\
\hline \pm 0.5 & 60 & 30 & 30 & - & - \\
\hline \pm 1.0 & 60 & 30 & 30 & 30 & 30 \\
\hline \pm 1.5 & $60^{*}$ & 30 & $120^{*}$ & 30 & 30 \\
\hline \pm 2.0 & 60 & 30 & 30 & - & - \\
\hline
\end{tabular}




\section{ANALYSIS AND RESULTS IN NOISE LEVEL}

\subsection{Methodology}

Experiments and their simulations carried out within the CORTEX work package 2 are to be compared in the frequency domain for validating the modelling tools [11]-[15]. The analysis of the experimental data in the frequency domain consists in calculating the Fourier transform of auto- and cross-correlation functions of respectively one or two detectors' signals. Without fuel rods' oscillation, auto- and cross-power spectral densities (APSD and CPSD) follow Equation (1) derived from point kinetics:

$$
G_{i j}(\omega)=\frac{2 C_{i} C_{j} D_{v}}{\beta^{2} F_{0}} \frac{1}{1+\left(\frac{\omega}{\alpha}\right)^{2}}+\sqrt{C_{i} C_{j}} \delta_{i j}
$$

where $C_{i}$ and $C_{j}$ are the count rates in the detectors, $D_{v}$ the Diven factor, $\beta$ the delayed neutron fraction, $F_{0}$ the total fission rate, $\omega=2 \pi \mathrm{f}$ the angular frequency, $\alpha$ the prompt decay constant, and $\delta_{i j}$ the Kronecker delta. In a CPSD, the latter term tends to zero because random coincidences of both detectors cancel out. In the case of a perturbation such as fuel rods' oscillation, it adds up in the PSD in the form of a peak at the perturbation frequency (see Figure 2). The quantities of interest for code validation of neutron noise solvers are the peak power, and the corresponding phase. [16]

The detectors' signals are first cleaned from unwanted contributions such as power drift, and normalized to their mean value. The coherence functions are computed to check the frequency consistency between detectors. PSD are computed using a Hann window of $2^{13}$ samples of $4 \mathrm{~ms}$ without overlapping, yielding a frequency resolution of around $0.03 \mathrm{~Hz}$. The uncertainty quantification is carried out by using bootstrapping with replacement on the time segments used for PSD estimate. An example of CPSD and peak power are presented in Figure 2 for a selected experiment performed with an oscillation amplitude of $\pm 1.5 \mathrm{~mm}$ and a frequency of $1 \mathrm{~Hz}$ that we will use in the rest of this article.

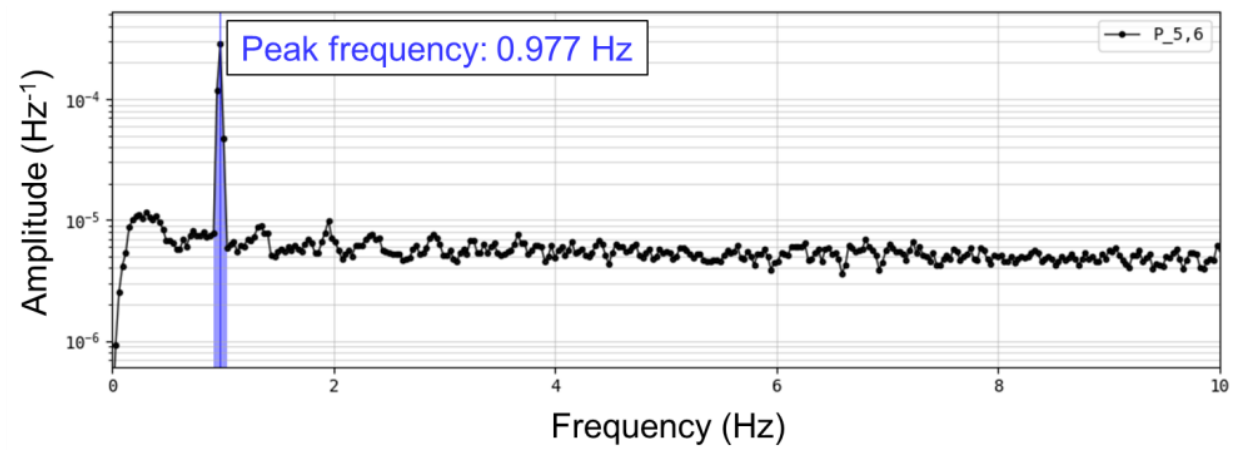

Figure 2. Example of a computed cross-power spectral density of detectors 5 and 6 , for an experiment with oscillation at $\pm \mathbf{1 . 5} \mathbf{~ m m} / \mathbf{1} \mathbf{~ H z}$. The peak power $P_{5-6}$, i.e. integrated amplitude, is highlighted in blue.

For validation purposes, the obtained powers are to be compared in relative, e.g. returned noise level by detector 6 with respect to detector 5. For each experiment, we compute all the following power ratios:

$$
R_{i j 5}(A, f)=\frac{P_{i-j}}{P_{5-j}}
$$

where $R_{i j 5}(A, f)$ is the ratio of power $P_{j-i}$ of the CPSD/APSD between detector $j$ and $i$, and power $P_{5-j}$ of the CPSD/APSD between detector $j$ and reference detector 5, at amplitude $A$ and frequency $f$. 
In Figure 3 left are represented the powers of all used detectors $j$ with respect to detectors $6\left(P_{6-j}\right.$, top) and 5 ( $P_{5-j}$, bottom) in blue. We observe that all the ratios $R_{6 j 5}$ return the same value (right) [5]. Discrepancies are observed for ratios using estimates from APSDs, which is expected as the APSD uncorrelated neutron noise also affects the power value under the peak. Using weighted averaging of all $R_{6 j 5}$, we calculate an optimized ratio of detector 6 to 5 , i.e. $R_{65}$. As an example, in Table II are presented all the ratios $R_{i j 5}$ for the experiment illustrated in Figures 2 and 3, as well as the weighted averaged ratios $R_{i 5}$. Ratios relying on APSDs are removed from the average (diagonal and line $j=5$ ), as well as outliers due to noise in the PSD (in red). It allows us to obtain a value with limited bias and reduced uncertainty, as well as observing the noise levels at each detector's position with respect to detector 5, taken as a reference. It allows as well the reconstruction of corrected absolute noise levels $\left\langle P_{i-5}\right\rangle$, by normalization to a selected detector (here 6).

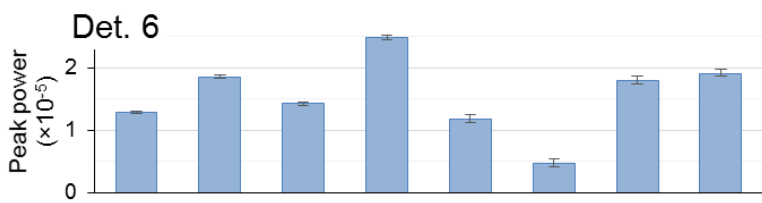

Det. 5

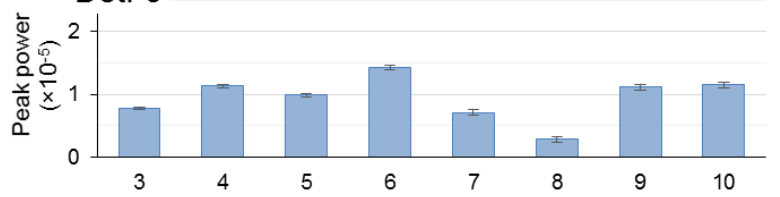

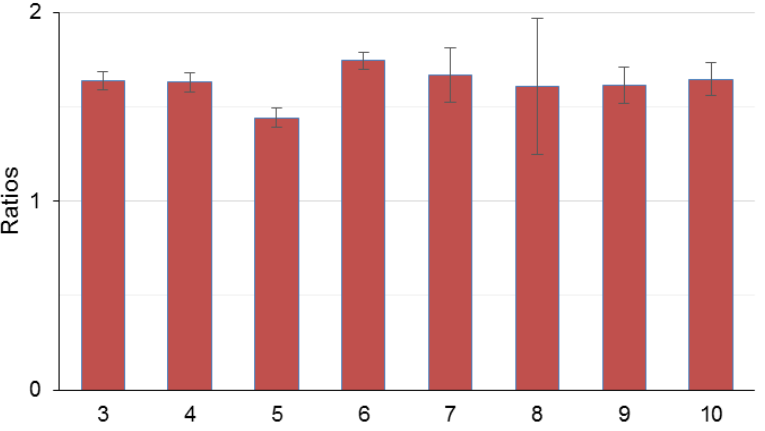

Figure 3. In blue, PSD peak power estimates of selected detectors 3 to 10 for an experiment $( \pm 1.5 \mathrm{~mm}$ $/ 1 \mathrm{~Hz}$ ) with respect to detectors 6 (top) and 5 (bottom), and comparatively with the ratios (red, right).

Table I. Calculated ratios $\boldsymbol{R}_{\boldsymbol{i j} 5}$ for the selected experiment $( \pm 1.5 \mathrm{~mm} / 1 \mathrm{~Hz})$ for all used detectors. Last lines indicate: weighted average ratios $R_{i 5}$, their deviation from $R_{55}$, and reduced uncertainties, and absolute peak power $\left\langle P_{i-5}\right\rangle$ reconstructed by normalization to detector 6 (i.e. $P_{5-6} / R_{65} \cdot R_{i 5}$ ).

\begin{tabular}{|c|c|c|c|c|c|c|c|c|}
\hline \multirow{2}{*}{ Detector $j$} & \multicolumn{9}{|c|}{ Ratios $R_{i j 5}$} \\
\cline { 2 - 9 } & \multicolumn{9}{|c|}{ Detector $i$} \\
\cline { 2 - 9 } & 3 & 4 & 5 & 6 & 7 & 8 & 9 & 10 \\
\hline 3 & 0.911 & 1.30 & 1 & 1.64 & 0.7 .97 & 0.317 & 1.26 & 1.32 \\
\hline 4 & 0.890 & 1.33 & 1 & 1.63 & 0.804 & 0.331 & 1.25 & 1.32 \\
\hline 5 & 0.789 & 1.15 & 1 & 1.44 & 0.718 & 0.288 & 1.12 & 1.16 \\
\hline 6 & 0.896 & 1.30 & 1 & 1.74 & 0.820 & 0.325 & 1.26 & 1.34 \\
\hline 7 & 0.874 & 1.29 & 1 & 1.65 & 3.61 & 1.96 & 1.18 & 1.26 \\
\hline 8 & 0.866 & 1.32 & 1 & 1.62 & 4.89 & 0 & 1.52 & 1.44 \\
\hline 9 & 0.883 & 1.28 & 1 & 1.61 & 0.756 & 0.389 & 3.03 & 1.30 \\
\hline 10 & 0.894 & 1.31 & 1 & 1.66 & 0.781 & 0.357 & 1.26 & 2.92 \\
\hline Ratios $R_{i 5}$ & 0.896 & 1.31 & 1 & 1.64 & 0.797 & 0.339 & 1.25 & 1.32 \\
\hline Deviation & $-10 \%$ & $31 \%$ & $0 \%$ & $64 \%$ & $-20 \%$ & $-66 \%$ & $25 \%$ & $32 \%$ \\
\hline Uncertainty & $1.5 \%$ & $1.6 \%$ & $1.5 \%$ & $1.8 \%$ & $3.2 \%$ & $7.1 \%$ & $2.5 \%$ & $2.3 \%$ \\
\hline$\left\langle P_{i-5}\right\rangle$ & $7.8210^{-6}$ & $1.1410^{-5}$ & $8.7210^{-6}$ & $1.4310^{-5}$ & $6.9510^{-6}$ & $2.9510^{-6}$ & $1.0910^{-5}$ & $1.1510^{-5}$ \\
\hline
\end{tabular}




\subsection{General behavior in amplitude and frequency}

The methodology presented in the previous section has been applied to all data. Hereafter we present the results in absolute peak power reconstructed for detector 5, i.e. $\left\langle P_{5-5}\right\rangle$. It is located on the opposite side of the core with respect to COLIBRI, and as such, it is expected to be most prone to the global effect of the perturbation. In Figure 4 (left, red), we observe the noise levels as a function of frequency for different oscillation amplitudes. As expected, the dependency in frequency is relatively stable with an increase at lower frequencies, as it is in the range below the cut-off frequency (around $25 \mathrm{~Hz}$ ) of the transfer function of CROCUS. In Figure 4 (right, green), we observe the increase in noise level corresponding to the increase in amplitude, for two oscillation frequencies. Again, as expected, it increases with amplitude, i.e. with the insertion of a larger perturbation in reactivity.
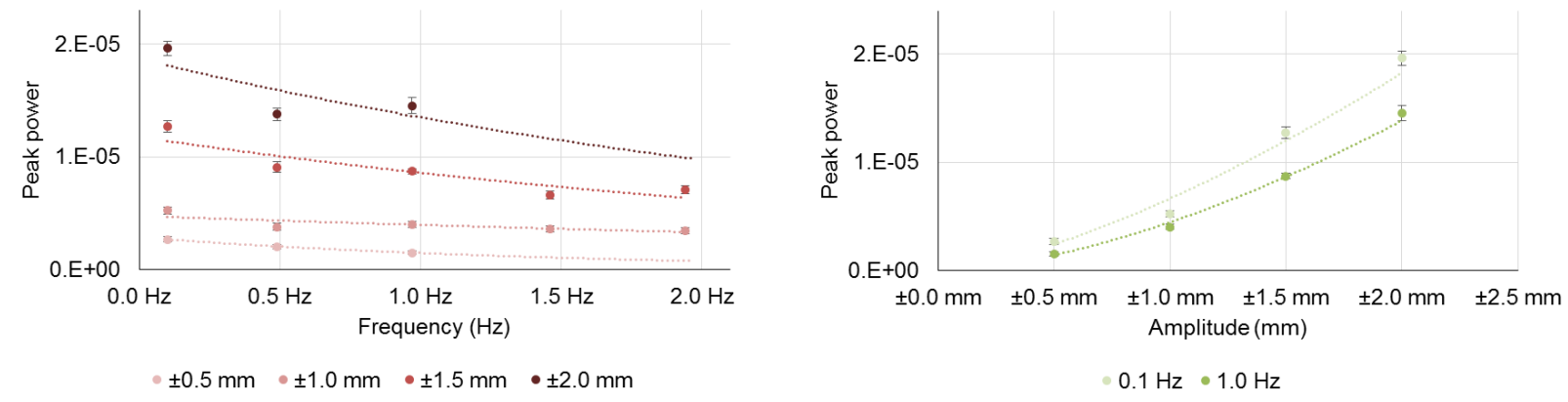

Figure 4. On the left, reconstructed absolute peak power estimates of detectors 5, i.e. $\left\langle P_{5-5}\right\rangle$, as a function of frequency for all amplitudes (left, red), and as a function of amplitude for two frequencies (right, green). The fitting curves are indicated for illustrative purposes.

\subsection{Spatial dependency}

At first, we focus on the comparison of noise levels in detector 6 as compared to the reference detector 5, i.e. the ratio $R_{65}$. The results are presented in percent of noise difference in Figure 5, as a function of frequency for two amplitudes (left, red), and as a function of amplitude for two frequencies (right, green). First, we observe that detector 6, located close to the perturbation, consistently returns higher noise levels than detector 5: it confirms that the noise levels are not simply following the flux map of CROCUS, but present a spatial dependency. The spatial dependency increases with frequency, and reaches a maximum at $\sim 80 \%$ around $\pm 1.5 \mathrm{~mm}$ in amplitude.
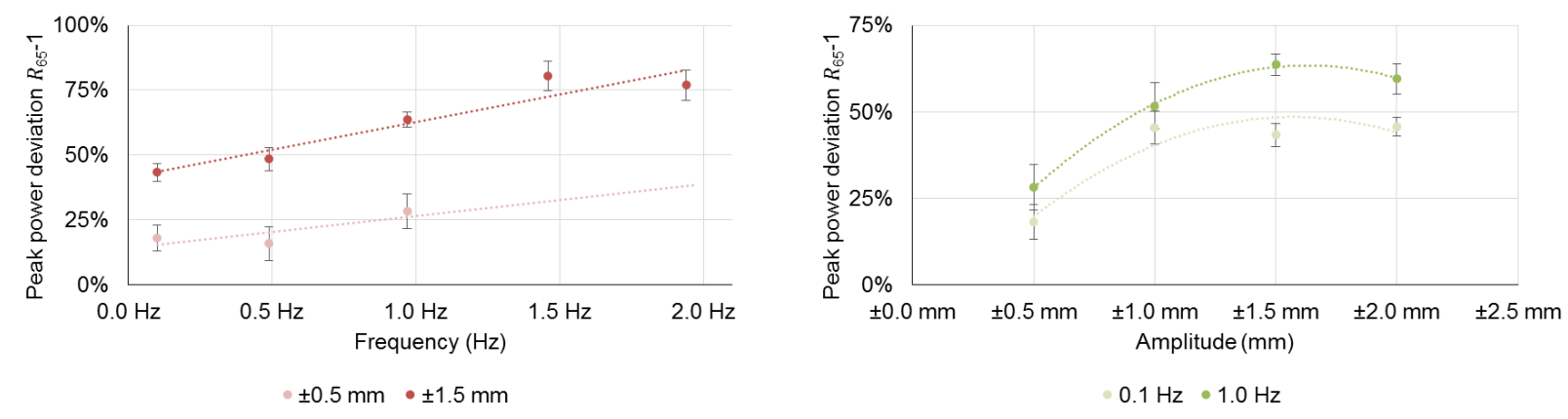

Figure 5. Deviation of noise level in detector 6 with respect to detector 5 , as a function of frequency for two amplitudes (left, red), and as a function of amplitude for two frequencies (right, green). The fitting curves are indicated for illustrative purposes. 
In Figure 6 are represented the deviations from the noise level in detector 5 for all detectors of an experiment at $\pm 1.5 \mathrm{~mm} / 0.1 \mathrm{~Hz}$, as a function of their distance to the perturbation (left), and on the CROCUS map (right). On the map, the deviations are coded in area and color (from blue for negative to red for positive), whereas the reference detector is white. For this experimental configuration, we observe a general increase of the noise level closer to the perturbation. The only outlier is detector 3 (blue), which consistently returns lower noise levels over all experiments. As this detector is an operation monitor, its unknown transfer function might be the reason for the assumed discrepancy. This case, as well as experimental results more generally, are compared to simulations and discussed in the twin article of this contribution [17].

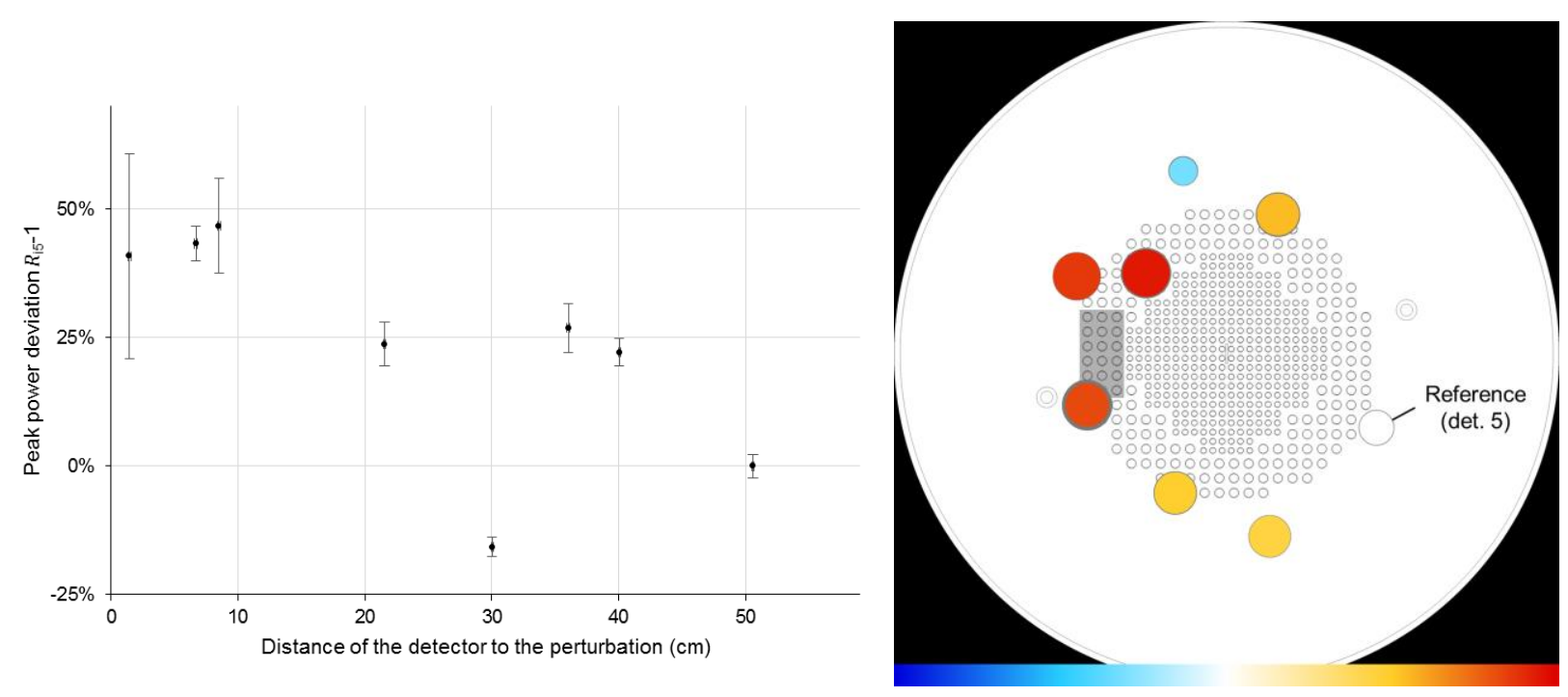

Figure 6. Relative difference in noise levels with respect to detector 5 , as a function of distance to the boundary of the perturbation (left), and on the CROCUS map (right). The COLIBRI perturbation area is represented by the grey rectangle. The differences are linearly coded in color from blue (min. $-50 \%)$ to red $(\max .+50 \%)$, with respect to the reference in white $(0 \%)$, as well as in disk area $(50 \%$ additional area corresponds to $+50 \%$ in noise). The thickness of the disks' rim corresponds to the uncertainty.

\section{CONCLUSIONS}

The first CORTEX noise campaign in CROCUS was carried out in 2018. Within the COLIBRI program, it consisted in experiments with a perturbation induced by a fuel rods oscillator. 18 fuel rods were oscillated between $\pm 0.5 \mathrm{~mm}$ and $\pm 2.0 \mathrm{~mm}$ around their central position in the lattice at a frequency range from $0.1 \mathrm{~Hz}$ to $2 \mathrm{~Hz}$. Signals from neutron detectors distributed in- and ex-core were recorded. A methodology was developed to obtain a robust estimate of the noise level in each detector, allowing a reduction of biases and uncertainties. The analysis of the experimental data demonstrates expected trends with regards to the global noise level as a function of the perturbation's amplitude and frequency. However, a significant spatial dependency of the noise was observed consistently over all detectors. Deviations of several tens of percent were characterized when compared to a reference detector, with limited uncertainty. These results are to be compared to simulation results, in noise level and phase, for the purpose of code validation. The second campaign in CROCUS, focusing on uncertainty reduction and spatial effects, was conducted in Oct. 2019.

\section{ACKNOWLEDGMENTS}

The authors thank the CROCUS operation team for its help and dedication, and the modelers of CORTEX for the fruitful discussions and the constant feedback. The research leading to these results has received funding from the Euratom research and training programme 2014-2018 under grant agreement No 754316. 


\section{REFERENCES}

1. C. Demazière, P. Vinai, M. Hursin, S. Kollias, and J. Herb, "Overview of the CORTEX project," in Proceedings of PHYSOR 2018, 2018, pp. 2971-2980.

2. V. Lamirand, A. Rais, S. Hübner, C. Lange, J. Pohlus, U. Paquee, C. Pohl, O. Pakari, P. Frajtag, D. Godat, M. Hursin, A. Laureau, G. Perret, C. Fiorina, and A. Pautz, "Neutron noise experiments in the AKR-2 and CROCUS reactors for the European project CORTEX," in ANIMMA 2019, 2019.

3. O. Pakari, V. Lamirand, G. Perret, P. Frajtag, and A. Pautz, "Kinetic Parameter Measurements in the CROCUS Reactor Using Current Mode Instrumentation," IEEE Trans. Nucl. Sci., vol. 65, no. 9, pp. 2456-2460, 2018.

4. M. Hursin, O. Pakari, G. Perret, P. Frajtag, V. Lamirand, I. Pazsit, V. Dykin, G. Por, H. Ferroukhi, and A. Pautz, "Measurement of the gas velocity in a water-air mixture in CROCUS by neutron noise technique," in International Conference on Mathematics and Computational Methods applied to Nuclear Science and Engineering (M\&C 2019), 2019.

5. V. Lamirand, A. Rais, S. Hübner, O. Pakari, M. Hursin, A. Laureau, C. Lange, J. Pohlus, U. Paquee, C. Pohl, P. Frajtag, D. Godat, G. Perret, C. Fiorina, and A. Pautz, "First results of neutron noise experiments in the AKR-2 and CROCUS reactors for the European project CORTEX," Submitt. to IEEE Trans. Nucl. Sci. Trans. Nucl. Sci., 2019.

6. V. Lamirand, P. Frajtag, D. Godat, M. Hursin, G. Perret, O. V. Pakari, A. Laureau, A. Rais, C. Fiorina, M. Chadwick, and A. Pautz, "The COLIBRI programme in CROCUS: development and licensing of a fuel rods oscillator," in RRFM/IGORR 2019, 2019.

7. J. M. Paratte, R. Früh, U. Kasemeyer, M. a. Kalugin, W. Timm, and R. Chawla, "A benchmark on the calculation of kinetic parameters based on reactivity effect experiments in the CROCUS reactor," Ann. Nucl. Energy, vol. 33, no. 8, pp. 739-748, May 2006.

8. U. Kasemeyer, R. Früh, J. M. Paratte, and R. Chawla, "Benchmark on Kinetic Parameters in the CROCUS Reactor," in International Reactor Physics Experiments Handbook (IRPhE), no. 4440, OECD, Ed. 2007, p. 94.

9. V. Lamirand, P. Frajtag, D. Godat, M. Hursin, G. Perret, O. Pakari, A. Rais, C. Fiorina, and A. Pautz, "The COLIBRI programme in CROCUS : characterisation of the fuel rods oscillator," in ANIMMA 2019, 2019.

10. A. Rais, V. Lamirand, O. Pakari, A. Laureau, J. Pohlus, C. Pohl, M. Hursin, C. Demazière, and A. Pautz, "Towards the validation of neutron noise simulators: qualification of data acquisition systems," in International Conference on Mathematics and Computational Methods applied to Nuclear Science and Engineering (M\&C 2019), 2019.

11. A. Mylonakis, P. Vinai, and C. Demazière, "Neutron noise modelling for nuclear reactor diagnostics," Proc. 27th Symp. Hell. Nucl. Phys. Soc., no. 754316, 2018.

12. A. Rouchon, A. Zoia, and R. Sanchez, "A new Monte Carlo method for neutron noise calculations in the frequency domain," Ann. Nucl. Energy, vol. 102, pp. 465-475, 2017.

13. T. Yamamoto, "Monte Carlo method with complex-valued weights for frequency domain analyses of neutron noise," Ann. Nucl. Energy, vol. 58, pp. 72-79, 2013.

14. A. Vidal-Ferràndiz, A. Carreño, D. Ginestar, C. Demazière, and G. Verdú, "A time and frequency domain analysis of the effect of vibrating fuel assemblies on the neutron noise," Ann. Nucl. Energy, 2019.

15. A. Gammicchia, S. Santandrea, I. Zmijarevic, R. Sanchez, Z. Stankovski, and S. Dulla, "A MOCbased neutron kinetics model for noise analysis," Submitt. to Ann. Nucl. Energy, 2019.

16. I. Pázsit and C. Demazière, "Noise Techniques in Nuclear Systems," in Handbook of Nuclear Engineering, D. G. Cacuci, Ed. Boston, MA: Springer US, 2010, pp. 1629-1737.

17. A. G. Mylonakis, C. Demazière, P. Vinai, V. Lamirand, A. Rais, O. Pakari, P. Frajtag, D. Godat, M. Hursin, G. Perret, A. Laureau, C. Fiorina, and A. Pautz, "CORE SIM+ simulations of COLIBRI fuel rods oscillation experiments and comparison with measurements," in Proceedings of PHYSOR 2020, 2020. 